\title{
Exzessive Palliativchirurgie
}

\author{
Karl Heinz Link ${ }^{a}$ Peter M. Schlag ${ }^{b}$ \\ a Chirurgisches Zentrum und Asklepios Tumortherapie Centrum Rhein-Main, Asklepios Paulinen Klinik, Wiesbaden, \\ ${ }^{\mathrm{b}}$ Klinik für Chirurgie und Chirurgische Onkologie, Charité, Universitätsmedizin Berlin, Deutschland
}

Nach schrittweisen Fortentwicklungen in der sogenannten «exzessiven Palliativchirurgie» sind mit Hilfe von interdisziplinären Therapiestrategien bei fortgeschrittenen primär nichtkurablen Tumorerkrankungen in ausgewählten Indikationen mittlerweile Ergebnisse zu erzielen, die denjenigen der rein palliativen konservativen Behandlung überlegen sind [1]. Die noch vor Jahren fast unmöglich erscheinenden Neuerungen werden in diesem Heft aktuell, informativ und verschiedene Indikationen betreffend dargestellt.

Die wesentlichen Fortschritte in der Behandlung gastrointestinaler und anderer solider Tumoren wurden bezüglich einer Prognoseverbesserung in den letzten zwei Dekaden durch die Kooperation von viszeralchirurgischen, gastroenterologischen und internistischen Onkologen sowie von Radioonkologen erzielt. Hervorzuheben sind die Resultate in der kurativen multimodalen Therapie von Kolon- und Rektumkarzinomen, neuerdings auch bei resektablen Pankreas- und Magenkarzinomen. Mit dem Ziel einer Optimierung der multimodalen Therapie wurde z.B. beim Kolonkarzinom im Stadium UICC III von einer der ersten deutschlandweiten interdisziplinären Arbeitsgruppen eine Steigerung der 5-Jahres-Überlebensrate von $49 \%$ (Chirurgie allein) über 59\% auf $72 \%$ (bester Therapiearm FOGT 1) erreicht. Solche Ergebnisse und die damit verbundenen Aktivitäten trugen mit dazu bei, den Weg zu interdisziplinären Kooperationen zu stärken.

Multimodale Behandlungsansätze bieten auch bei primär chirurgisch-onkologisch nichtkurablen Primärtumoren oder Metastasen heutzutage eine Verbesserung der Prognose und sogar Heilungschancen. Zu Beginn der 1990er Jahre konnten wir z.B. über die Möglichkeit berichten, inoperable kolorektale Lebermetastasen nach Ansprechen auf Chemotherapie sekundär mit kurativem Ansatz zu resezieren - in einer Zeit, zu der diese Patienten entweder nur symptomatisch oder mit einer nur bedingt wirksamen systemischen Chemotherapie rein palliativ behandelt wurden. Derartige Konzepte stießen bei «konservativen» Chirurgen und Onkologen zunächst auf Skepsis und weckten Kritik. Mit der Zeit haben sich die Akzeptanz dieses neuen Therapieansatzes und die zur Durchführung notwendigen Kooperationsstrukturen positiv gewandelt. Zahlreiche Literaturberichte, auch aus den eigenen Arbeitsgruppen, haben Ende der 1990er Jahre den Benefit der Resektion primär irresektabler Lebermetastasen nach gutem Ansprechen auf die mittlerweile effektivere systemische Chemotherapie herausgestellt. Palliative Chirurgie allein mit Teilresektion von symptomatischen Metastasen oder primären Lebertumoren bietet bis auf die Ausnahme neuroendokriner hormonell aktiver Lebermetastasen oder akut rupturierter Tumoren keinen Benefit [2]. Bei symptomatischen, fortgeschrittenen hepatobiliären und pankreatischen (Primär-)Tumoren kann dagegen die rein palliative Chirurgie unter akzeptabler Mortalität teilweise effektiver sein als die mangels wirksamerer therapeutischer Alternativen praktizierte konservative symptomorientierte Behandlung [3]. Umgekehrt kann man bei synchron fortgeschritten metastasierten asymptomatischen Kolon- und Rektumkarzinompatienten auf die palliative Primärtumorresektion bzw. Stomaanlage verzichten und ohne Verlust der Überlebensqualität - primär die systemische Chemotherapie einsetzen, die bei gutem Ansprechen sogar eine komplette sekundäre Resektion von Primärtumor und Metastasen und somit auch ein Langzeitüberleben ermöglichen kann [4].

Noch 1990 wurde die Meinung vertreten, dass die Resektion (resektabler!) kolorektaler Lebermetastasen für den Patienten keinen Vorteil hat. Diese Auffassung wird z.B. bei Kolonoder Rektumkarzinomen heute auch für Patienten mit isolierter Peritonealkarzinose vertreten. In diesem Erkrankungsstadium werden in der Routine bisher nur rein palliativchirurgische und möglichst einfache chirurgische Verfahren eingesetzt. Phase-III- und große Beobachtungsstudien zeichnen auch hier eine neue Entwicklung vor. In spezialisierten Zen-

\begin{tabular}{|c|c|}
\hline KARGER & (C) 2007 S. Karger GmbH, Freiburg \\
\hline $\begin{array}{l}\text { Fax +49 } 7614520714 \\
\text { E-mail Information@Karger.de } \\
\text { www.karger.com }\end{array}$ & $\begin{array}{l}\text { Accessible online at: } \\
\text { www.karger.com/cga }\end{array}$ \\
\hline
\end{tabular}

Prof. Dr. Dr. h.c. Karl Heinz Link

Chirurgisches Zentrum und Asklepios Tumortherapie Centrum Rhein-Main Asklepios Paulinen Klinik

Geisenheimer Straße 10, 65197 Wiesbaden, Deutschland

Tel. +49 611 8472-431, Fax -399

E-mail k-h.link@asklepios.com 
tren wird bei zirkumskripter Peritonealkarzinose (vor allem beim Kolon-, Magen- und Ovarialkarzinom) das Konzept der zytoreduktiven Chirurgie mit gleichzeitiger intraoperativer hyperthermer intraperitonealer Chemotherapie (HIPEC) oder einer intensivierten perioperativen intraperitonealen Chemotherapie jeweils kombiniert mit einer additiven systemischen i.v. Chemotherapie verfolgt [4-6]. Für selektierte Patienten lassen sich hiermit die Langzeitüberlebenschancen im Vergleich zur bisherigen konservativen Standardbehandlung deutlich verbessern. Allerdings darf die potentielle Morbidität der chirurgisch-multimodalen Therapie nicht außer Acht gelassen werden. Um diese so gering wie möglich zu halten, sollte eine solche exzessive Palliativchirurgie spezialisierten
«High-volume»-Zentren mit langjähriger Erfahrung vorbehalten sein, die den Stellenwert des Verfahrens in prospektiven, randomisierten Studien weiter untersuchen.

Es besteht kein Zweifel, dass exzessive Palliativchirurgie nur im engen interdisziplinären Zusammenspiel von Gastroenterologen, Medizinischen Onkologen und Radioonkologen sinnvoll betrieben werden kann [7]. Es ist ein besonderes Anliegen der Chirurgische Arbeitsgemeinschaft Onkologie der Deutschen Gesellschaft für Visceralchirurgie (CAO-V), diese Zusammenarbeit zu pflegen und weiter auszubauen. Vom Stahl allein - althergebracht geführt - sind in der viszeralen Onkologie weniger positive Entwicklungen zu erwarten als von einer «Gastrointestinalen Chirurgie Interdisziplinär».

\section{Literatur}

1 Hempen HG, Raab H.-R: Beckeneviszeration beim rezidivierten und lokal weit fortgeschrittenen Rektumkarzinom. Chir Gastroenterol 2007;23(4):DOI 10.1159/000109423.

2 Lang H: Erweiterte multimodale Leberresektionen bei primären und sekundären Lebertumoren. Chir Gastroenterol 2007;23(4):DOI 10.1159/000109996.

3 Bahra M, Jacob D, Thelen A, Neumann UP: Oberbaucheviszeration beim fortgeschrittenen hepatopankreatischen Karzinom. Chir Gastroenterol 2007;23(4):DOI 10.1159/000109413.
4 Weber T, Link KH: Radikale Chirurgie bei primär metastasierten kolorektalen Karzinomen. Chir Gastroenterol 2007;23(4):DOI 10.1159/000110482. 5 Glockzin G, Schlitt HJ, Piso P: Zytoreduktive Chirurgie und hypertherme intraperitoneale Chemotherapie bei der Behandlung des kolorektalen Karzinoms. Chir Gastroenterol 2007;23(4):DOI $\underline{10.1159 / 000109411}$
6 Gretschel S, Schlag, PM: Multiviszerale Resektion als Baustein eines multimodalen Behandlungskonzeptes beim lokal fortgeschrittenen oder peritoneal metastasierten Magenkarzinom. Chir Gastroenterol 2007;23(4):DOI 10.1159/000111986.

7 Beller S, Schlag PM: Hat die palliative Resektion bei gastrointestinalen Tumoren noch einen Stellenwert? Chirurg 2006;77(3):219-226. 